\title{
Valve-sparing excision of aortic valve papillary fibroelastoma
}

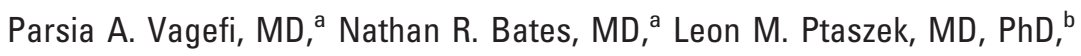 \\ Mark S. Adams, RDCS, ${ }^{\mathrm{c}}$ and Jennifer D. Walker, $\mathrm{MD}^{\mathrm{a}}$
}
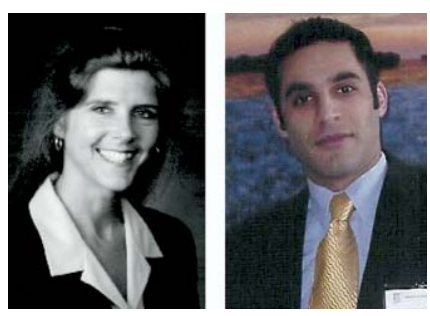

Supplemental material is available online.

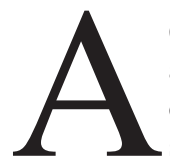

65-year-old woman with a history of hypertension and anxiety presented to an outside hospital after the sudden onset of feeling a rightward tilt. The patient also described loss of her right superior temporal visual field for approximately 45 minutes and short-term memory difficulties at that time but denied any other symptoms. The patient's physical examination was unremarkable. She underwent a computed tomographic scan of her head that was normal, and she was discharged home. That same evening, the patient began complaining of nausea and bifrontal headaches and on returning to the outside hospital was admitted. The results of a lumbar puncture were negative. A subsequent magnetic resonance image (MRI) of her brain demonstrated evidence of small emboli to the left anterior and posterior circulation involving the occipital and parietal lobes. The results of bilateral carotid duplex studies were negative. By report, a transthoracic echocardiogram revealed two $1-\mathrm{cm}$ vegetations on the aortic valve, with no other valve pathology and no patent foramen ovale. The patient was started on antibiotics for presumed endocarditis.

Three days after her initial presentation, the patient was transferred to our institution for further care. Repeat transthoracic echocardiography and subsequent transesophageal echocardiography demonstrated a mass on the right coronary cusp of the aortic valve. This mass measured $11 \times 9 \times 10 \mathrm{~mm}$ and appeared attached by a stalk to the right coronary cusp. Computed tomographic angiography was performed to evaluate the patient's coronary circulation. The aortic valve mass was visualized, but no evidence of luminal narrowing was found (Figure 1, $A$ and $B$ ). The patient underwent a cardiac MRI for further characterization of the lesion. This demonstrated a mass in the right sinus of Valsalva with attachment to the aortic surface of the right coronary cusp of the aortic valve close to the commissure with the noncoronary cusp (Figure 1, C). The lesion measured $11 \times 11$ $\mathrm{mm}$. There was no convincing evidence of extension through or

From the Divisions of Cardiac Surgery, ${ }^{\mathrm{a}}$ Cardiology, ${ }^{\mathrm{b}}$ and Cardiothoracic Anesthesia, ${ }^{\mathrm{c}}$ Massachusetts General Hospital, Harvard Medical School, Boston, Mass.

Received for publication Dec 1, 2005; revisions received Dec 22, 2005; accepted for publication Dec 30, 2005.

Address for reprints: Jennifer D. Walker, MD, Massachusetts General Hospital, 55 Fruit St, EDR 105, Boston, MA 02114-2696 (E-mail: jdwalker@ partners.org).

J Thorac Cardiovasc Surg 2006;131:1177-8

$0022-5223 / \$ 32.00$

Copyright $\odot 2006$ by The American Association for Thoracic Surgery

doi:10.1016/j.jtcvs.2005.12.053
Drs Walker and Vagefi (left to right).

outside the wall of the right sinus of Valsalva. Given the patient's presentation and radiographic findings, it was believed that the patient's presentation was caused by embolization from a cardiac neoplasm. The patient was started on intravenous heparin and clopidogrel (Plavix, Bristol-Myers Squibb, New York, N.Y.; the patient had an aspirin allergy), and the patient was subsequently referred to the cardiac surgical service.

Ten days after admission to our institution, the patient was brought to the operating room. On opening the aorta, the aortic valve was inspected and revealed a mass that was fleshy and brown with a grape-like cluster appearance (Figure E1, $A$ ). The mass was excised sharply from the valve cusp, avoiding injury to the cusp while ensuring complete surgical excision (Figure E1, B). The patient was subsequently separated from bypass without difficulty. An intraoperative transesophageal echocardiogram after excision demonstrated a completely competent valve with no aortic insufficiency and a normal aorta (Figure 2). The chest was closed, and the patient was transported to the cardiac intensive care unit in stable condition. On postoperative day 1, the patient was transferred out of the intensive care unit to the step-down unit. Subsequently, she was discharged home on postoperative day 5. Final pathology demonstrated a papillary fibroelastoma (Figure E1, C).

Although rare and benign tumors, papillary fibroelastomas are the second most common primary cardiac tumor in adults. ${ }^{1}$ The valvular surfaces comprise the predominant location of this tumor, with the most common being the aortic valve, followed by the mitral valve. Thirty percent of papillary fibroelastomas are diagnosed incidentally in asymptomatic individuals (by means of echocardiography, during cardiac surgery, or at autopsy). The most common clinical presentation, usually caused by embolization of the tumor or thrombus, has been reported as being either stroke or transient ischemic attack, followed by angina, myocardial infarction, sudden death, heart failure, syncope or presyncope, and systemic or pulmonary embolic events. ${ }^{2}$ Recurrence after complete surgical resection has not been observed. ${ }^{3}$ A recently published retrospective review, the largest surgical series to date describing treatment and outcome of patients with cardiac papillary fibroelastoma, demonstrated an $83 \%$ rate of shave excision without the need for valvular repair or replacement. ${ }^{4}$ Of the 88 patients in this study, $52 \%$ had disease involving the aortic valve.

We report here a patient with the sudden onset of neurologic symptoms caused by embolization from an aortic valve papillary fibroelastoma who underwent successful aortic valve-sparing resection of the tumor. Preoperative cardiac MRI and echocardiography were used to assess the aortic valve and the associated mass before complete excision. Because the patient was deemed at high risk for further embolization with standard coronary angiography, a preoperative cardiac computed tomography angiography was performed as a noninvasive method to rule out the concomitant presence of coronary artery disease. $^{5}$ 
A

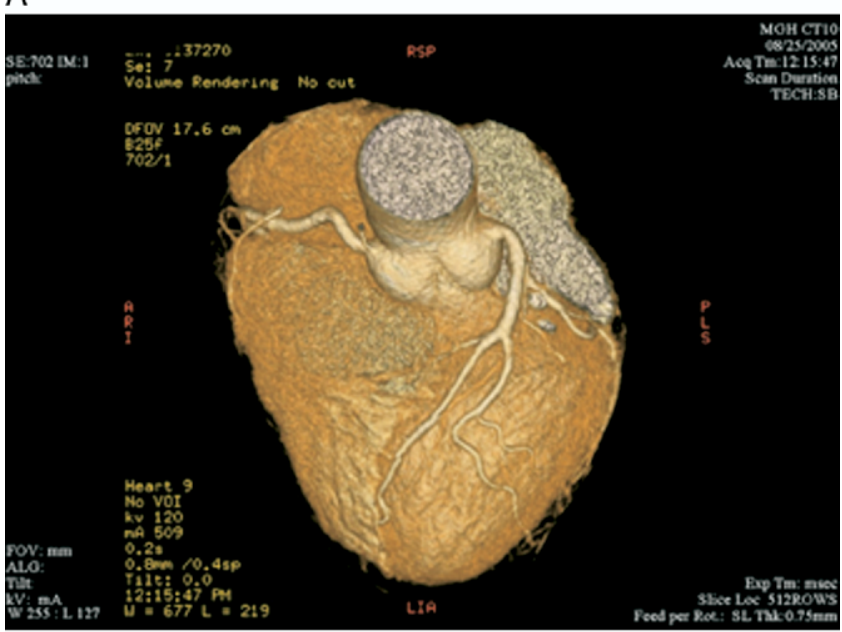

B

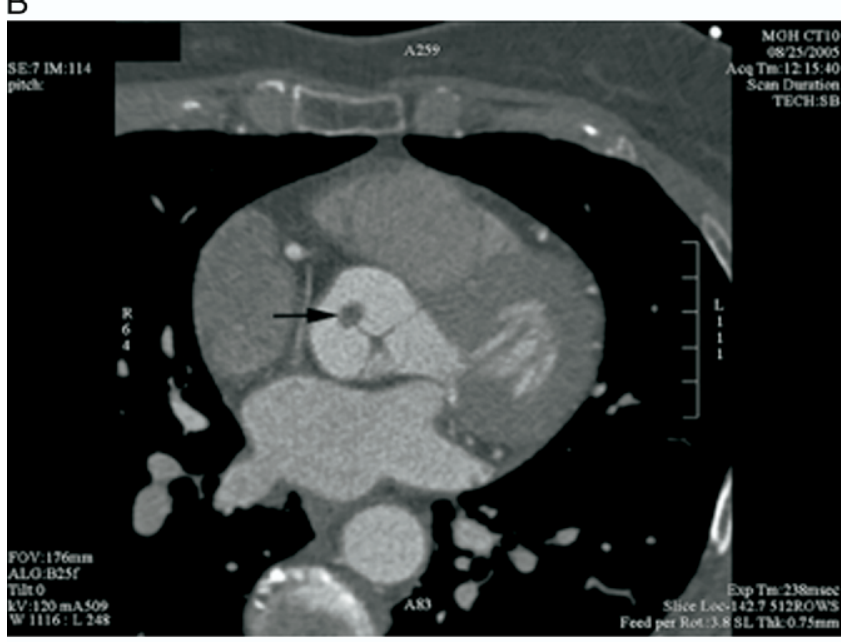

C

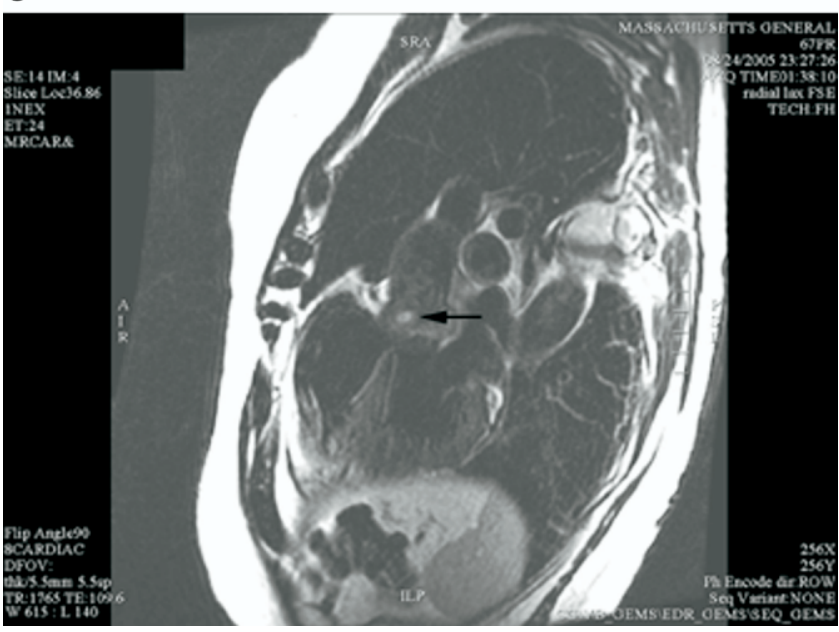

Figure 1. A cardiac computed tomography angiography was obtained and did not demonstrate any evidence of coronary artery disease (A); however, a small mass lesion arising from the coronary cusp was seen (B, arrow). A cardiac MRI T2 image from the breath-hold fast spin-echo series demonstrates the known mass lesion in the right sinus of Valsalva with attachment to the right coronary cusp of the aortic valve close to the commissure with the noncoronary cusp (C, arrow).
A

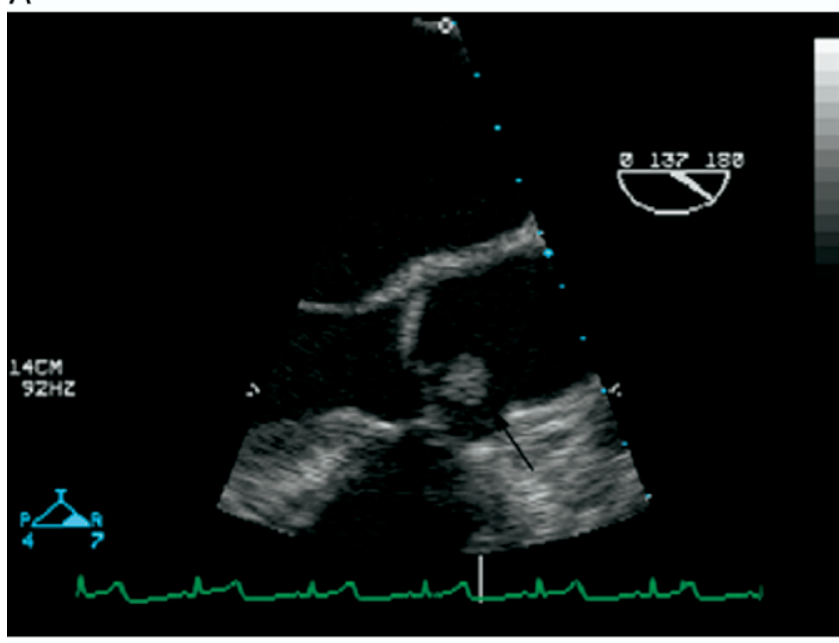

B

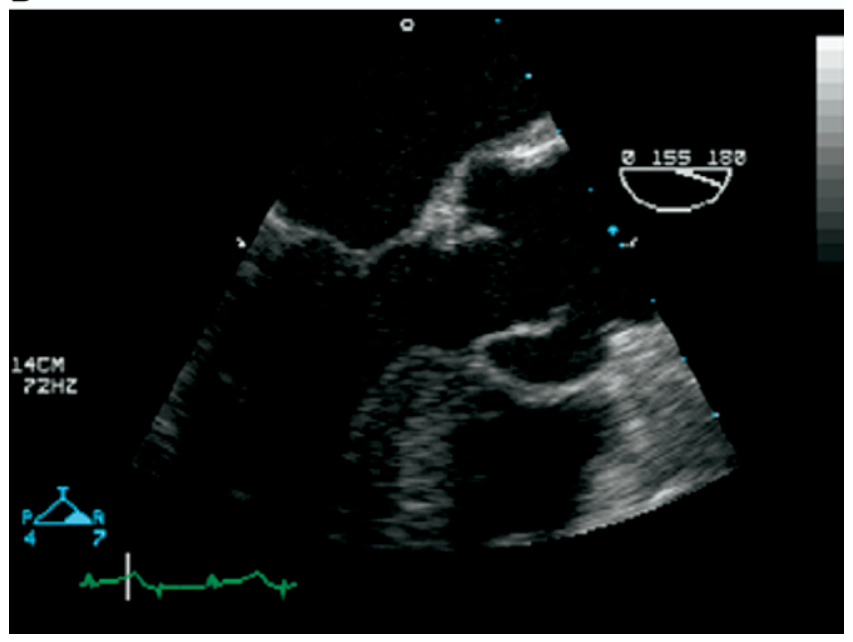

Figure 2. Intraoperative transesophageal echocardiogram demonstrating an aortic valve mass before valve-sparing excision ( $A$, arrow) and subsequently after tumor excision (B).

\section{References}

1. Gowda RM, Khan IA, Nair CK, Mehta NJ, Vasavada BC, Sacchi TJ Cardiac papillary fibroelastoma: a comprehensive analysis of 725 cases. Am Heart J. 2003;146:404-10.

2. Sun JP, Asher CR, Yang XS, Cheng GG, Scalia GM, Massed AG, et al. Clinical and echocardiographic characteristics of papillary fibroelastomas: a retrospective and prospective study in 162 patients. Circulation. 2001;103:2687-93.

3. Grandmougin D, Fayad G, Moukassa D, Decoene C, Abolmaali K, Bodart JC, et al. Cardiac valve papillary fibroelastomas: clinical, histological and immunohistochemical studies and a physiopathogenic hypothesis. J Heart Valve Dis. 2000;9:832-41.

4. Ngaage DL, Mullany CJ, Daly RC, Dearani JA, Edwards WD, Tazelaar $\mathrm{HD}$, et al. Surgical treatment of cardiac papillary fibroelastoma: a single center experience with eighty-eight patients. Ann Thorac Surg. 2005;80: $1712-8$.

5. Schoepf UJ, Becker CR, Ohnesorge BM, Yucel EK. CT of coronary artery disease. Radiology. 2004;232:18-37.

1178 The Journal of Thoracic and Cardiovascular Surgery • May 2006 


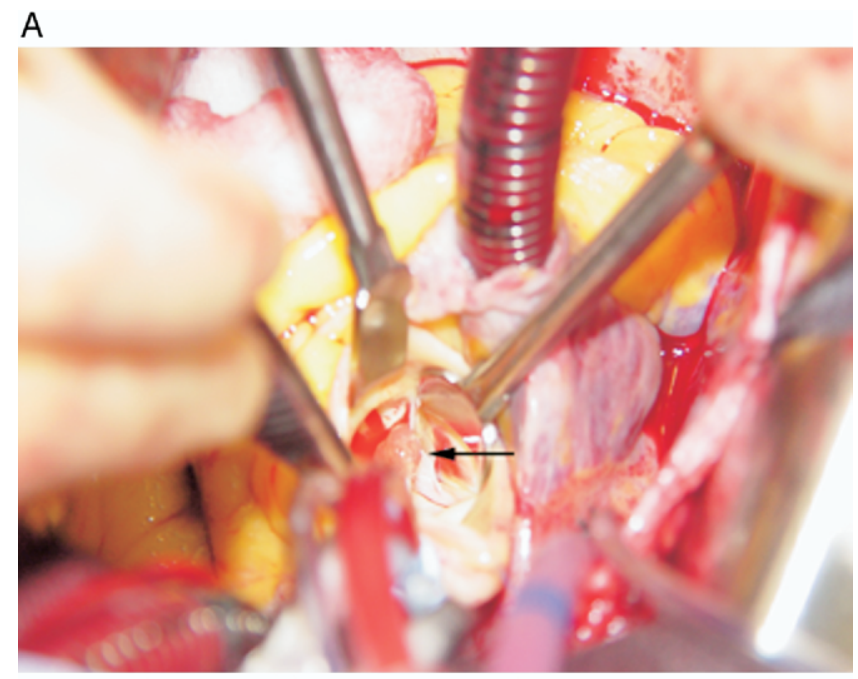

B

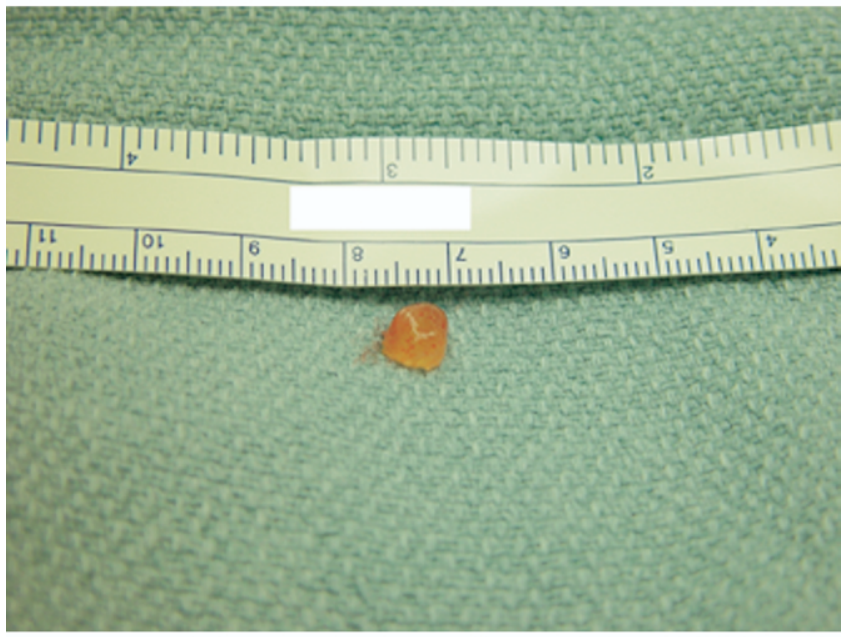

C

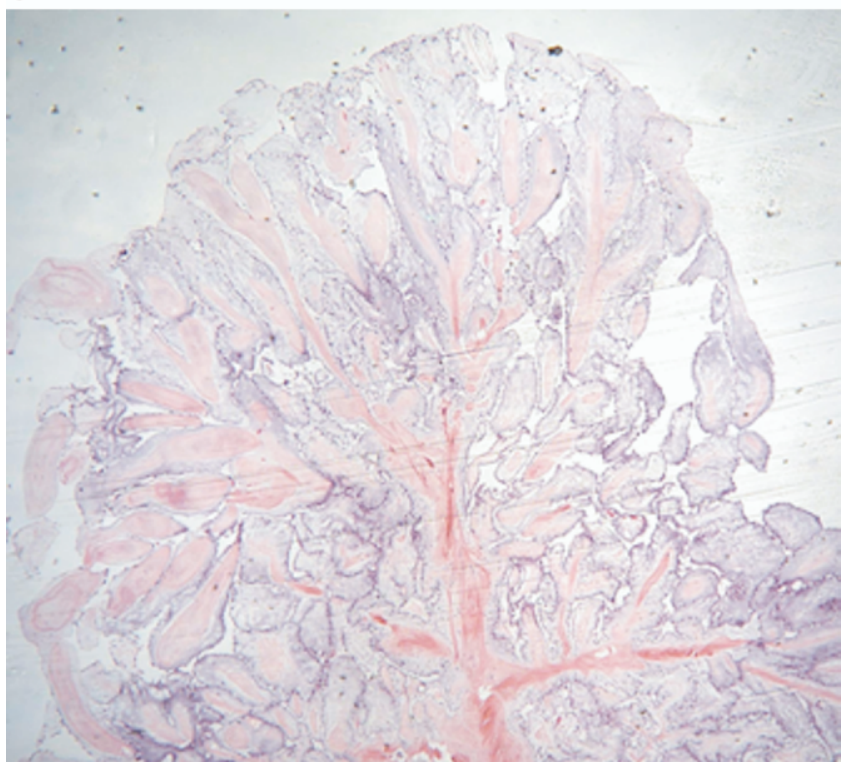

Figure E1. A, Intraoperative photo of the aortic valve mass (arrow). B, Gross specimen after resection off of the aortic valve. C, Histology of aortic valve mass $(2 x)$ demonstrating papillary fibroelastoma. 